\title{
Non-invasive Pressure Reactivity Index Using Doppler Systolic Flow Parameters: A Pilot Analysis
}

\author{
Frederick A. Zeiler, ${ }^{1-3}$ Peter Smielewski, ${ }^{4}$ Andrew Stevens, ${ }^{1}$ Marek Czosnyka ${ }^{4,5}$, David K. Menon, ${ }^{1}$ Ari \\ Ercole $^{1}$
}

1. Division of Anaesthesia, Addenbrooke's Hospital, University of Cambridge, Cambridge, UK 2. Department of Surgery, Rady Faculty of Health Sciences, University of Manitoba, Winnipeg, MB, Canada

3. Clinician Investigator Program, Rady Faculty of Health Sciences, University of Manitoba, Winnipeg, $\mathrm{MB}$, Canada

4. Division of Neurosurgery, Department of Clinical Neurosciences, Addenbrooke's Hospital, University of Cambridge, Cambridge, UK

5. Institute of Electronic Systems, Warsaw University of Technology, Warsaw, Poland

\section{Corresponding Author:}

Frederick A. Zeiler BSc MD FRCSC (Neurosurgery)

Assistant Professor

Department of Surgery

Rady Faculty of Health Sciences

University of Manitoba

Winnipeg, MB, Canada

Email: umzeiler@myumanitoba.ca

Contributing Authors:

Peter Smielewski PhD

Section of Brain Physics

Division of Neurosurgery

University of Cambridge

Email: ps10011@cam.ac.uk

Andrew R. Stevens MBChB BMedSc

Division of Anaesthesia

University of Cambridge

Email: a.stevens@doctors.org.uk 
Marek Czosnyka PhD

Professor of Brain Physics

Section of Brain Physics

Division of Neurosurgery

University of Cambridge

Cambridge, UK

CB2 OQQ

Email: mc141@medschl.cam.ac.uk

David K. Menon MD PhD FRCP FRCA FFICM FMedSci

Head, Division of Anaesthesia, University of Cambridge

Honorary Consultant, Neurosciences Critical Care Unit, Addenbrooke's Hospital

Professorial Fellow, Queens' College, Cambridge

Senior Investigator, National Institute for Health Research, UK

Email:dkm13@cam.ac.uk

Ari Ercole MD PhD FRCA FFICM

Consultant in Intensive Care Medicine

Division of Anaesthesia

University of Cambridge

Email: ae105@cam.ac.uk 


\section{Abstract:}

The goal was to predict pressure reactivity index (PRx) using non-invasive transcranial Doppler (TCD) based indices of cerebrovascular reactivity, systolic flow index (Sx_a) and mean flow index (Mx_a). Continuous extended duration time series recordings of middle cerebral artery cerebral blood flow velocity (CBFV) were obtained using robotic TCD in parallel with direct intracranial pressure (ICP). PRx, Sx_a and Mx_a were derived from high frequency archived signals. Using time-series techniques, autoregressive integrative moving average (ARIMA) structure of PRx was determined and embedded in the following linear mixed effects (LME) models of PRx: PRx Sx_a and PRx Sx_a + Mx_a. Using 80\% of the recorded patient data, the LME models were created and trained. Model superiority was assessed via Akaike information criterion (AIC), Bayesian information criterion (BIC) and log-likelihood (LL). The superior two models were then used to predict PRx using the remaining $20 \%$ of the signal data. Predicted and observed PRx were compared via Pearson correlation, linear models and Bland-Altman (BA) analysis. Ten patients had 3 to 4 hours of continuous uninterrupted ICP and TCD data and were used for this pilot analysis. Optimal ARIMA structure for PRx was determined to be $(2,0,2)$, and this was embedded in all LME models. The top two LME models of PRx were determined to be: PRx Sx_a and PRx Sx_a + Mx_a. Estimated and observed PRx values from both models were strongly correlated ( $r>0.9 ; p<0.0001$ for both), with acceptable agreement on BA analysis. Predicted PRx using these two models was also moderately correlated with observed PRx, with acceptable agreement $(r=0.797$, $p=0.006 ; r=0.763, p=0.011 ;$ respectively). With application of ARIMA and LME modelling, it is possible to predict PRx using non-invasive TCD measures. This is the first and preliminary attempts at doing so. Much further work is required. Keywords: autoregulation, brain injury, TBI, TCD, time series

\section{Introduction:}


Pressure reactivity index (PRx) is considered the "gold standard" of continuous cerebrovascular reactivity monitoring after traumatic brain injury (TBI). ${ }^{1,2}$ Derived from the moving correlation coefficient between slow-wave fluctuations in intra-cranial pressure (ICP) and mean arterial pressure (MAP), PRx provides a continuously updating assessments of cerebral autoregulation, with positive values indicative of impaired cerebrovascular reactivity. ${ }^{1,3}$ Numerous observational studies have linked persistently positive PRx values with poor global outcome in TBI, with well-defined critical thresholds associated with morbidity and mortality. ${ }^{4,5}$ Furthermore, PRx is one of only a few continuous indices of cerebrovascular reactivity to have been validated for assessing the lower limit of autoregulation in an experimental model. ${ }^{6}$ Finally, current applications of continuously updating indices of cerebrovascular reactivity have focused on the derivation of "personalized" cerebral perfusion pressure (CPP) targets (also referred to as CPP optimum), utilizing PRx. ${ }^{7,8}$ However, the requirement of invasive ICP measurements for PRx calculation is a limitation of the technique.

Various other continuous indices of cerebrovascular reactivity exist based on other invasive and noninvasive monitoring devices employed after TBI. ${ }^{3,9}$ In particular, non-invasive transcranial Doppler (TCD) can be utilized to derive flow-based indices. ${ }^{4,10}$ Recent multi-variate co-variance analysis has confirmed a close association between non-invasive TCD derived systolic flow index (Sx_a) - the moving correlation between systolic flow velocity (FVs) and MAP) and invasively derived ICP based indices, including PRx. ${ }^{11,12}$ Furthermore, time series linear modelling techniques highlight this strong relationship between PRx and Sx_a, while providing evidence to support the ability to estimate PRx accurately using this noninvasive TCD index. ${ }^{13}$

The next natural step would be to attempt the prediction of PRx using this non-invasive TCD measure. This has never been attempted before, given complexity of analysis and limitations surrounding acquisition of continuous longer uninterrupted TCD recordings. The goal of this study was to outline the 
first experience at predicting PRx using non-invasive Sx_a, derived from extended duration robotic TCD recordings.

\section{Methods:}

\section{$\underline{\text { Patient Population }}$}

The data utilized in this retrospective analysis was part of a prospective observational study conducted over a 6-month period within our unit (December 2017- May 2018). All patients suffered from moderate to severe TBI and were admitted to the neurosciences critical care unit (NCCU) at Addenbrooke's Hospital, Cambridge. Patients were intubated and sedated given the severity of their TBI. Invasive ICP monitoring was conducted in accordance with the Brain Trauma Foundation (BTF) guidelines. Therapeutic measures were directed at maintaining ICP less than $20 \mathrm{mmHg}$ and CPP greater than $60 \mathrm{mmHg}$ (datum at the tragus).

TCD monitoring is a part of standard intermittent cerebral monitoring within the NCCU. The application of the newer robotic TCD device (see description below) was therefore in alignment with our usual care, negating the need for formal direct or proxy consent. All data related to patient admission demographics and high frequency digital signals from monitoring devices were collected in an entirely anonymous format and ethical approval for research using anonymous data acquired as part of clinical practice is not required under UK regulations. 


\section{Signal Acquisition}

Various signals were obtained through a combination of invasive and non-invasive methods. Arterial blood pressure (ABP) was obtained through either radial or femoral arterial lines connected to pressure transducers (Baxter Healthcare Corp. CardioVascular Group, Irvine, CA). ICP was acquired via an intraparenchymal strain gauge probe (Codman ICP MicroSensor; Codman \& Shurtleff Inc., Raynham, MA). Finally, TCD assessment of MCA CBFV was conducted via a robotic TCD system, the Delica EMS 9D (Delica, Shenzhen, China, www.delicasz.com). This system allows for continuous extended duration recording of MCA CBFV, using robotically controlled TCD probes, with automated correction algorithms for probe shift. To our knowledge, this is the first study on the application of extended duration TCD acquisition via a robotic system in critically ill TBI patients.

This study aimed to record 3 to 4 hours of continuous data from all devices simultaneously, given the previous work from our group on inter-index relationships focused on recording durations of only 0.5 to 1-hour duration due to limitation of conventional TCD. As such, this data set also proved to be ideal for complex time series modelling and analysis. Only patients with 3 or more continuous, uninterrupted, ICP and TCD recordings were utilized for this study. Thus, only a sub-population of the group from the original study were utilized for this analysis. Figure 1 displays an example of the recording set up, including triple bolt, near infrared spectroscopy and robotic TCD.

\section{*Figure 1 here}




\section{Signal Processing}

Signals were recorded using digital data transfer, sampled at frequency of $100 \mathrm{Hertz}(\mathrm{Hz})$ or higher (depending on the modality), using ICM+ software (Cambridge Enterprise Ltd, Cambridge, UK, http://icmplus.neurosurg.cam.ac.uk). Signal artifact were removed using a combination of manual and semi-automated methods within ICM+ prior to further processing or analysis.

Post-acquisition processing of the above signals was conducted using ICM+ software. CPP was determined using the formula: $C P P=$ MAP $-I C P . T C D$ signal was analyzed from the right side in the majority of the patients given right frontal placement of ICP monitors. The only exception to this is when we were unable to obtain quality TCD on the right due to poor windows for TCD.

Systolic flow velocity (FVs) was determined by calculating the maximum flow velocity (FV) over a 1.5 second window, updated every second. Diastolic flow velocity (FVd) was calculated using the minimum FV over a 1.5 second window, updated every second. Mean flow velocity (FVm) was calculated using average FV over a 10 second window, updated every 10 seconds (ie. without data overlap).

Ten second moving averages (updated every 10 seconds to avoid data overlap) were calculated for all recorded signals: ICP, ABP (which produced MAP), CPP, FVm, FVs and FVd. These non-overlapping 10second moving average values allow focus on slow-wave fluctuations in signals by decimating the signal frequency to $\sim 0.1 \mathrm{~Hz}$.

Cerebrovascular reactivity indices were derived in a similar fashion across modalities. PRx: A moving Pearson correlation coefficient was calculated between ICP and MAP using 30 consecutive 10 second windows (ie. five minutes of data), updated every minute. Similar to our previous work on non-invasive estimation of PRx using Doppler measures, two non-invasive TCD based indices were also derived: Mx-a 
(the correlation between FVm and MAP) and Sx-a (the correlation between FVs and MAP). Diastolic flow index (Dx-a) was not evaluated in this study, given our previous work documenting poor time series and linear mixed effects (LME) model performance in relation to PRx. ${ }^{13}$

Data for this analysis were provided in the form of a minute by minute time trends of the parameters of interest for each patient. This was extracted from ICM+ in to comma separated values (CSV) datasets, which were collated into one continuous data sheet (compiled from all patients).

\section{Statistical Analysis}

Similar statistical modelling approach as seen in our previous work for time series data and LME model creation were followed with this data set, and almost identical statistical description to his work will be found below. ${ }^{13}$ Minute-by-minute time series data was utilized for the entirety of the analysis described below. Statistical significance was set at an alpha of less than 0.05 . All statistical analysis was conducted using R statistical software (R Core Team (2016). R: A language and environment for statistical computing. R Foundation for Statistical Computing, Vienna, Austria. URL https://www.R-project.org/). The following packages were utilized during the analysis: tseries, forecast, lubridate and Ime4.

The statistical methods sections to follow will outline the techniques employed to: $[A]$ estimate the autocorrelative structure of PRx in time series; $[\mathrm{B}]$ create an accurate model estimating PRx using noninvasive TCD indices of cerebrovascular reactivity via application of linear mixed effects (LME) modelling (with embedded PRx autocorrelative error structure); [C] assess the correlation and agreement between model based estimated PRx and the observed PRx value; and [D] predict PRx using the derived LME models and estimated PRx time series data. For LME model creation/training we utilized the $1^{\text {st }}$ $80 \%$ of the data for each patient, with the remaining $20 \%$ utilized for the prediction of PRx using the LME models. 


\section{Autocorrelative Structure of PRx}

Prior to being able to model PRx using TCD based indices, it was necessary to determine the autocorrelation structure of PRx. We used Box-Jenkin's autoregressive integrative moving average (ARIMA) modelling PRx to determine: the autoregressive structure of order " $p$ ", the differencing factor or order " $d$ ", and the moving average component of order " $q$ "; commonly denoted " $(p, d, q)$ ". The autoregressive structure refers to the dependence of PRx at time $t$ (denoted PRxt) on previous measures of PRx (ie. called "lags"), say at time $t-1$ (ie. $P R x_{t-1}$ ), and so forth (ie. say to PRx $x_{t-p}$ ), with the order " $p$ " indicating how many previous PRx measures PRxt is dependent on. Stationarity is defined here as the presence of a stable variance, autocorrelative structure and mean over time. Stationarity can be introduced by differencing previous PRx measures from current measures, thus removing trending structure from a time series, and allowing further modelling to occur. The differencing order " $\mathrm{d}$ " refers to how many previous terms should be included in the differencing process. Finally, the moving average term refers to the need to include the error in the ARIMA model at time $t$ (ie. $\varepsilon_{t}$ ) as well as " $q$ " previous error terms (ie. $\varepsilon_{t-1}, i=1 . . q$ ). Assuming stationarity (ie. no " $d$ " order), the ARIMA model folds to a general ARMA model that can be represented by the following formula:

$$
\mathrm{PRX}_{\mathrm{t}}=\mathrm{C}+\varepsilon_{\mathrm{t}}+\sum_{i=1}^{p} \varphi P R x_{\mathrm{t}-\mathrm{i}}+\sum_{i=1}^{q} \theta \varepsilon \mathrm{t} \mathrm{-i}
$$

Where: $\mathrm{PRx} x_{\mathrm{t}}=\mathrm{PRx}$ at time $t, \mathrm{PRx} x_{t-i}=\mathrm{PRx}$ at time $t-i, \varepsilon_{t}=$ error at time $t, \varepsilon_{t-i}=$ error at time $t-i, c=$ constant, $\varphi$ and $\vartheta$ are parameters at time $t-i, p=$ autoregressive order, and $q=$ moving average order.

The following process was conducted on all patient recordings, in order to derive the optimal ARIMA structure for PRx time series. This would provide insight into the approximate best ARIMA structure for future LME models. 
First, data had already been artifact cleared and had a 10-second moving average filter applied to the data, leading to some data smoothing (as described above in the signal processing section). Thus, our initial step for the ARIMA modelling focused on determining stationarity of the signal. This was assessed, and confirmed, using three methods. First, we assessed the autocorrelation function (ACF) correlogram for PRx, looking for a rapid decline in significant lags, indicating a stationary signal. Second, we employed the Augmented Dickey Fuller (ADF) test to assess for stationarity. Third, we attempted seasonal decomposition using the like-named function in R for each PRx time series, which employs locally weighted scatterplot smoothing (LOWESS) to identify seasonal and trend components to a time series. All above processes confirmed stationarity within our patient examples.

Second, the autoregressive structure of PRx was assessed using the ACF correlograms and partial autocorrelation function (PACF) correlograms. ACF correlograms were assessed to see how many previous consecutive terms (ie. "lags") PRx may be dependent upon. Similarly, the PACF correlograms were assessed to see how many non-consecutive previous lags, PRx may be dependent upon. Significant level on ACF/PACF correlograms is set at a correlation level of $+/-\left(2 / N^{1 / 2}\right)$, where $N=$ sample size. We then ran sequential ARMA models for PRx by varying the order " $p$ " from 0 to 3 , while also varying the moving average order " $q$ " from 0 to 3 . Given our analysis for stationarity confirmed a stationary signal within our 10 patient examples, we fixed the differencing order " $d$ " at 0 . In doing so we generated 16 separate ARMA models for PRx within each patient. Model superiority was assessed by Akaike Information Criterion (AIC) and Log-Likelihood (LL), with the lowest AIC and highest LL indicating the best ARMA model for PRx. In addition, model superiority was assessed via residuals, model ACF and PACF correlograms, with an adequate model represented by random residuals, and ACF/PACF failing to display any lags reaching significance. 


\section{LME Modelling of PRx Using TCD Derived Indices}

LME modelling was conducted on the entire patient population. LME modelling involved various fixed linear models, and a random component introduced into the intercept and independent variable coefficient (based on individual patient). We embedded the PRx ARIMA structure within the LME models (based on the ARIMA modelling results analysis above). This analysis was done on the full data set, deriving LME models for each patient as well as for the entire population. The following LME models were assessed, initially with random intercept only (stratified by patient), as above: PRx Sx_a, PRx Mx_a, and PRx Sx_a + Mx_a. All models were corrected using maximum likelihood estimation method. Adequacy of the LME model was assessed via QQ plots and the residuals distribution plot, with linear shape to the QQ plots and normally distributed residuals confirming validity of the model.

Models were compared using AIC, Bayesian Information Criterion (BIC), LL and analysis of variance (ANOVA) testing. Superior models were attributed to the lowest AIC, lowest BIC and highest LL. Significance differences between models were assessed by ANOVA testing, with a threshold for significance set at a $p<0.05$. The top 2 superior LME models were reported in detail, with a final assessment of model adequacy through ACF/PACF plots of the model residuals, observing for a minimal number of significant lags which decay rapidly.

Generalized fixed effects models were also created based on the top two LME models. However, these models performed poorly, with substantially inferior AIC, BIC and LL values. In addition, these general fixed effects models maintained continuous significant lags in the residuals, further indicating poor modelling of PRx. Hence, these models will not be discussed further. 


\section{Observed versus Estimated PRx}

We assessed the correlation between the observed (minute-by-minute) PRx values in our population versus those estimated from our optimal two LME models using Pearson correlation coefficient. We then produced linear regression plots between observed and estimated PRx for the best two LME models, using grand mean data (ie. mean value per patient). Finally, Bland-Altman plots were produced to assess agreement between the observed and estimated PRx values, using grand mean Fisher transformed data (ie. Fisher transform applied to both observed and estimated PRx).

\section{Predicting PRx}

We predicted PRx based on the top two LME models from the above discussed methods. Using the LME models themselves and the remaining $20 \%$ patient data not used in LME creation, we derived predicted PRx (pPRx) values from observed Sx-a and Mx-a values within this data subset. The predicted values were then compared to the actual observed PRx values during this period using Pearson correlation, linear modelling and Bland-Altman analysis.

\section{Results:}

\section{a. Patient Demographics}

A total of 10 patients with moderate/severe TBI had sufficient quality TCD signals (ie. at least $3-4$ hours duration and uninterrupted). The mean age for this population was $34.5+/-17.0$ years, with 8 patients being male. The median admission GCS was 7 (IQR 4 to 8), with median admission GCS motor score of 4 (IQR 2 to 5). The mean duration of ICP/TCD recording was $223.2+/-38.4$ minutes. Only $80 \%$ of the total 
recording duration for each patient was utilized for model formation and training, with the remaining $20 \%$ reserved for predictive testing.

\section{b. Building the Model to Estimate PRx}

\section{ARIMA Modelling of PRx}

In all 10 patients the ARIMA structure of PRx was investigated in order to determine the appropriate structure for future LME modelling of the entire population. Upon inspection of the ACF/PACF plots, ADF test results and seasonal decomposition techniques, it was determined that no significant trend or seasonality were present in any of the 10 patient recordings. Thus, no differencing order " $d$ " was introduced. Next, sequential ARMA models were produced for each patient, varying the autoregressive order " $p$ " and moving average order " $q$ ", from 0 to 3. Across all patients the optimal ARMA model for PRx was found to be $(2,0,2)$, based on the principle of parsimony, and the lowest AIC and highest LL. Figure 2 displays the ACF and PACF plots of PRx for one patient with 4 hours of continuous recording, demonstrating a rapid decay in significant lags (implying stationarity). Figure 3 shows the residuals for the ARMA model for PRx in the same patient, with an ARMA structure of $(2,0,2)$. This figure demonstrates a lack of significant lags on ACF and PACF plots, with randomly distributed residuals, confirming adequacy of the chosen model.

\footnotetext{
*Figure 2 and 3 here
} 


\section{c. Model Development and Accuracy Assessment}

\section{LME Modelling of PRx Using TCD Indices}

Using the $(2,0,2)$ PRx ARMA structure identified within the individual patients, various LME models were produced, embedding the PRx ARMA structure within them. Table 1 displays the model characteristics for those LME models derived from Sx_a and Mx_a, introducing random effects by patient into the intercept and coefficients. Model superiority was confirmed via ANOVA testing, with the lowest AIC/BIC and highest LL, indicating superiority. The top two LME models were: PRx $\sim S x \_a$ $(A I C=-1564.957, B I C=-1510.159, L L=792.4786)$ and PRx $\sim S x \_a+M x \_a(A I C=-1597.345, B I C=-$ $1520.627, \mathrm{LL}=812.6726)$; with random effects by patient introduced into both the coefficients and intercept. The $\mathrm{QQ}$ and residual density plots for these top two LME models can be seen in Appendix A, displaying normally distributed residuals, indicating model adequacy. The ACF and PACF plots for these two models can also be found in Appendix A, displaying acceptable rapid decay of significant lags.

\section{Population Based Estimation of PRx Using Sx-a and Mx-a}

Using the top two LME models described above, PRx was estimated using the available Sx_a and Mx_a measures in the training data set. Grand mean values were calculated per patient and plotted against the observed PRx values from the data. A strong linear relationship was seen between estimated and observed PRx using both LME models. Figure 4 displays estimated versus observed PRx plots for each model. The PRx Sx_a model displayed a correlation between estimated and observed values of $0.998(95 \% \mathrm{Cl}=0.990-0.999 ; p<0.0001)$, while the PRx $\sim$ Sx_a + Mx_a model had a correlation between estimated and observed PRx values of $0.997(95 \% \mathrm{Cl}=0.988-0.999$; $p<0.0001)$. Bland-Altman analysis on Fisher transformed results displayed acceptable agreement, 
with slight underestimation bias in the estimated PRx for both models. This bias in the Bland-

Altman plots was seen in our previous work as well. ${ }^{13}$ Appendix B contains the results of the BlandAltman analysis comparing the estimated to observed PRx for both LME models.

\section{*Table 1 here}

\section{*Figure 4 here}

\section{d. Predicting PRx Using Non-Invasive TCD Parameters}

Using the top two LME models derived above, we proceeded to predict PRx (pPRx) using the $20 \%$ of data not used in model construction/training. Each patient had $20 \%$ of their recording data excluded from the prior model formation/training, each with ICP and TCD derived variables, amounting to 30 to 60 minutes of minute-by-minute data per patient. For each LME model, the Sx_a and Mx_a values from this new data were entered into the models to derive pPRx. Grand mean values were then calculated per patient. For the model PRx Sx_a, the correlation between predicted and observed PRx values was $0.797(95 \% \mathrm{Cl}=0.336-0.949 ; p=0.006)$. Similarly, for the model PRx $\sim$ Sx_a + Mx_a, the correlation between predicted and observed PRx was $0.763(95 \% \mathrm{Cl}=0.258-0.941 ; p=0.011)$. Predicted and observed PRx values displayed a linear association, though not 1:1. Figure 5 displays the predicted versus observed PRx plots for the top two LME models. Bland-Altman analysis of Fisher transformed data demonstrated acceptable agreement between predicted and observed PRx values, with similar 
underestimation of the predicted PRx values as seen in the training data previously. All Bland-Altman results for comparing pPRx to observed PRx can be found in Appendix C.

*Figure 5 here

\section{Discussion:}

Through the application of time-series ARMA and LME modelling in this pilot study we have been able to describe, for the first time, the prediction of pressure reactivity index PRx using non-invasive TCD derived cerebral autoregulation measures (in this case Sx_a and $\mathrm{Mx}$ _a). Some important aspects of this preliminary pilot work require highlighting.

First, through the application of ARMA modelling of PRx, and LME modelling of PRx using TCD measures, in this unique cohort of patients with extended duration continuous TCD recordings, we have been able to produced LME models that accurately estimate observed PRx. This is similar to our prior retrospective work in a large TB population with TCD. ${ }^{13}$ Further, the superior two models from this current cohort were of similar AMRA and mixed-effects structure to those discovered in the prior work, as confirmed through the principle of parsimony. This provides some validation of the previous work, and also provides some evidence to support these models regardless of the duration of TCD recording analyzed. In addition, the bias on Bland-Altman analysis comparing observed to estimated PRx displayed the same underestimation bias seen in our previous work, with acceptable agreement.

Second, as with our previous work, ${ }^{13}$ the general fixed effects versions of our top two models performed poorly in estimating PRx, with continuous significant autocorrelation in the model residuals. As mentioned within the methods section, these models were subsequently not reported further. This 
again confirms patient-by-patient heterogeneity, limiting the extrapolation of this work to other general TBI populations.

Third, the current, and previous, ${ }^{13}$ ARMA works demonstrate the strong relationship between measures of CBV (ie. ICP or PRx) and CBF (ie. CBFV or Sx_a/Mx_a). This strong association between measures of $\mathrm{CBV}$ and $\mathrm{CBF}$ is important to emphasize. PRx, believed to be a measure of cerebrovascular pressure reactivity, relies on the correlation between vasogenic slow-wave fluctuations in ICP and MAP. ICP in this instance is considered a surrogate measure of pulsatile CBV. Thus, PRx is measuring changes in CBV in response to changes in MAP, with the corresponding correlation representing cerebral pressure autoregulation and having been validated to measure the lower-limit of autoregulation in various experimental models. ${ }^{6,14,15}$ Whereas, the TCD based cerebrovascular reactivity indices (ie. Sx_a and Mx_a) are based on the correlation between slow-wave fluctuations in CBFV and MAP. Thus, TCD based indices may be considered to be closer measures of flow than those based on non-CBF/CBFV measures. The main limiting factor for TCD measures is the labor-intensive nature inherent with classic TCD devices leading to short and interrupted recordings. Thus, the application of TCD for regular continuous monitoring of cerebrovascular reactivity has been limited, with no experimental studies in existence validating them as measures actual of pressure autoregulation. Through demonstrating the strong link between PRx and Sx_a/Mx_a, we have demonstrated that TCD based flow measures are close in relation to the validated pressure autoregulation measure PRx However, it also must be acknowledged this relationship between volume and flow is not necessarily the same across all patients, as exemplified by the poor performance of general fixed effects models and the need of LME modelling demonstrating clearly that the flow/volume relationship varies from patient to patient.

Fourth, we demonstrated for the first time in the literature the ability to estimate PRx using noninvasive TCD surrogates. Comparing pPRx to the observed PRx values in the top two models, the correlation is of moderate strength with a linear relationship between the two and acceptable 
agreement on Bland-Altman analysis. However, a similar underestimation bias for pPRx is present on Bland-Altman analysis. Further, the relationship between pPRx and the observed PRx was not 1:1, indicating the prediction is not perfect. This is despite having very strong correlations between the estimated PRx and observed PRx during the model training phase. This potentially suggests model over fitting during the training, though it must be acknowledged the current work is mainly a proof of concept and pilot analysis. Much further work is required to optimize the prediction models.

Fifth, for the first time in the literature we successfully applied the emerging robotic TCD technology for extended duration recording in critically ill TBI patients.

Finally, this work is based on only 10 patients and is entirely preliminary with results that are not generalizable at this time. Thus, this type of modelling and prediction of PRx should not be conducted outside of a research setting. Much further work is required for validation.

\section{$\underline{\text { Limitations }}$}

As with our previous work in this area, the patient population was heterogeneous in terms of age, intracranial injury patterns and therapies directed at ICP/CPP goals. These heterogeneities could impact signal fluctuations and the results obtained for the time series modelling conducted. Though in comparison to our larger time series work in $\mathrm{TBI}$, the results of the modelling within this preliminary pilot study were similar, as described above.

Second, our patient numbers were small, at only 10. For the purpose of this type of analysis we chose only to look at patients with 3 to 4 hours of completely uninterrupted ICP and TCD recordings and thus the dataset, whilst small, is of exceptional length for routine clinical recordings. The requirement for interrupted continuous TCD is a significant limitation with the described modelling, as conventional TCD 
is currently heavily limited by artifact and signal loss. The application of the robotic TCD mitigated this in our study, however this technology is relatively new and not without its own limitations, including patient eligibility (ie. no decompressive craniectomy, no cervical spine immobilization devices, etc.), because of the robotic probe relatively bulky design. Furthermore, even with the application of robotic $T C D$, it remains difficult to obtain continuous extended duration recordings given patient motion, bedside procedures and transport for routine and emergent neuro-imaging. As robotic and automated TCD technology improves, we expect to be able to obtain extended duration uninterrupted recordings throughout a patient's ICU stay. Thus, even though the current results are limited given patient numbers, they provide the platform for future applications once technology catches up with the demands of this type of modelling/prediction.

Third, the ARIMA structure highlighted for PRx, and the LME models within this study may not be widely applied outside this population. This was also mentioned in our previous publication on this topic. There exists the potential for patient specific ARIMA structures, and thus the models described in our studies should not be applied clinically. Furthermore, as mentioned above, the general fixed effects versions of our top two models performed poorly in the estimation of PRx, resulting in persistently significant residual lags. This also suggests significant patient-by-patient heterogeneity, negating the extrapolation of these results to other general TBI populations at this time. Further work on PRx, amongst other physiologic measures in $\mathrm{TBI}$, is required in larger patient populations in order to determine the exact high frequency time series behavior.

Finally, the statistical methodology employed within this study is quite complex. Thus, the wide spread applicability of these techniques is currently limited. However, with the increasing availability of realtime bedside computing software such constraints are becoming less important as even mathematically sophisticated models can be handled automatically allowing for a much more user-friendly application of such techniques. Such functions will automate much of the analysis described in our works on time 
series, requiring limited user input. This will hopefully bring this type of work to the wider clinical world for future multi-center validation studies.

\section{Conclusions:}

Through the application of ARMA and LME modelling, it is possible to estimate PRx using non-invasive TCD measures, such as Sx_a and Mx_a. This is the first preliminary attempts at doing so. Much further work is required prior to application within a clinical setting, as this the current work should be considered experimental at this time.

Disclosures: FAZ has received salary support for dedicated research time, during which this project was partially completed. Such salary support came from: the Cambridge Commonwealth Trust Scholarship, the Royal College of Surgeons of Canada - Harry S. Morton Travelling Fellowship in Surgery and the University of Manitoba Clinician Investigator Program.

PS has financial interest in a part of licensing fee for ICM+ software (Cambridge Enterprise Ltd, UK).

MC has financial interest in a part of licensing fee for ICM+ software (Cambridge Enterprise Ltd, UK).

DKM has consultancy agreements and/or research collaborations with GlaxoSmithKline Ltd; Ornim Medical; Shire Medical Ltd; Calico Inc.; Pfizer Ltd; Pressura Ltd; Glide Pharma Ltd; and NeuroTraumaSciences LLC. 
Acknowledgments: This work was made possible through salary support through the Cambridge Commonwealth Trust Scholarship, the Royal College of Surgeons of Canada - Harry S. Morton Travelling Fellowship in Surgery and the University of Manitoba Clinician Investigator Program.

These studies were supported by National Institute for Healthcare Research (NIHR, UK) through the Acute Brain Injury and Repair theme of the Cambridge NIHR Biomedical Research Centre and an NIHR Senior Investigator Award to DKM. Authors were also supported by a European Union Framework Program 7 grant (CENTER-TBI; Grant Agreement No. 602150)

Statement of Authorship: FAZ was responsible for concept, data collection, data analysis, statistical methodology manuscript composition. AS was responsible for data collection and manuscript composition. PS, MC, DKM and AE were responsible for manuscript writing and editing.

\section{References:}

1. Czosnyka, M., Smielewski, P., Kirkpatrick, P., Laing, R.J., Menon, D., and Pickard, J.D. (1997). Continuous assessment of the cerebral vasomotor reactivity in head injury. Neurosurgery $41,11-$ 17; discussion 17-19.

2. Czosnyka, M., Miller, C., and Participants in the International Multidisciplinary Consensus Conference on Multimodality Monitoring. (2014). Monitoring of cerebral autoregulation. Neurocrit Care 21 Suppl 2, S95-102.

3. Zeiler, F.A., Donnelly, J., Calviello, L., Smielewski, P., Menon, D.K., and Czosnyka, M. (2017). Pressure Autoregulation Measurement Techniques in Adult Traumatic Brain Injury, Part II: A Scoping Review of Continuous Methods. J. Neurotrauma 34, 3224-3237. 
4. Sorrentino, E., Diedler, J., Kasprowicz, M., Budohoski, K.P., Haubrich, C., Smielewski, P., Outtrim, J.G., Manktelow, A., Hutchinson, P.J., Pickard, J.D., Menon, D.K., and Czosnyka, M. (2012). Critical thresholds for cerebrovascular reactivity after traumatic brain injury. Neurocrit Care 16, 258-266.

5. Zeiler, F.A., Donnelly, J., Smieleweski, P., Menon, D., Hutchinson, P.J., and Czosnyka, M. (2018). Critical Thresholds of ICP Derived Continuous Cerebrovascular Reactivity Indices for outcome prediction in Non-Craniectomized TBI Patients: PRx, PAx and RAC. J. Neurotrauma 160, 1315-1324.

6. Brady, K.M., Lee, J.K., Kibler, K.K., Easley, R.B., Koehler, R.C., and Shaffner, D.H. (2008). Continuous measurement of autoregulation by spontaneous fluctuations in cerebral perfusion pressure: comparison of 3 methods. Stroke 39, 2531-2537.

7. Howells, T., Smielewski, P., Donnelly, J., Czosnyka, M., Hutchinson, P.J.A., Menon, D.K., Enblad, P., and Aries, M.J.H. (2018). Optimal Cerebral Perfusion Pressure in Centers With Different Treatment Protocols. Crit. Care Med. 46, e235-e241.

8. Needham, E., McFadyen, C., Newcombe, V., Synnot, A.J., Czosnyka, M., and Menon, D. (2017). Cerebral Perfusion Pressure Targets Individualized to Pressure-Reactivity Index in Moderate to Severe Traumatic Brain Injury: A Systematic Review. J. Neurotrauma 34, 963-970.

9. Zeiler, F.A., Donnelly, J., Calviello, L., Menon, D.K., Smielewski, P., and Czosnyka, M. (2017). Pressure Autoregulation Measurement Techniques in Adult Traumatic Brain Injury, Part I: A Scoping Review of Intermittent/Semi-Intermittent Methods. J. Neurotrauma 34, 3207-3223.

10. Budohoski, K.P., Reinhard, M., Aries, M.J.H., Czosnyka, Z., Smielewski, P., Pickard, J.D., Kirkpatrick, P.J., and Czosnyka, M. (2012). Monitoring cerebral autoregulation after head injury. Which component of transcranial Doppler flow velocity is optimal? Neurocrit Care 17, 211-218.

11. Zeiler, F.A., Donnelly, J., Menon, D.K., Smielewski, P., Zweifel, C., Brady, K., and Czosnyka, M. (2017). Continuous Autoregulatory Indices Derived from Multi-Modal Monitoring: Each One Is Not Like the Other. J. Neurotrauma 34, 3070-3080.

12. Zeiler, F.A., Cardim, D., Donnelly, J., Menon, D.K., Czosnyka, M., and Smielewski, P. (2018). Transcranial Doppler Systolic Flow Index and ICP-Derived Cerebrovascular Reactivity Indices in Traumatic Brain Injury. J. Neurotrauma 35, 314-322.

13. Zeiler, F.A., Smieleweski, P., Donnelly, J., Czosnyka, M., Menon, D., and Ercole, A. (2018). Estimating Pressure Reactivity Index Using Non-Invasive Doppler Based Systolic Flow Index. J. Neurotrauma .

14. Zeiler, F.A., Lee, J.K., Smielewski, P., Czosnyka, M., and Brady, K. (2018). Validation of ICP derived cerebrovascular reactivity indices against the lower limit of autoregulation, Part II: experimental model of arterial hypotension. J Neurotrauma Epub Ahead of Print.

15. Zeiler, F.A., Donnelly, J., Calviello, L., Lee, J.K., Smielewski, P., Brady, K., Kim, D.J., and Czosnyka, M. (2018). Validation of pressure reactivity and pulse amplitude indices against the lower limit of autoregulation, Part I: experimental intra-cranial hypertension. J Neurotrauma In Press. 
Table 1: LME Models with PRx $(2,0,2)$ ARIMA Structure - Entire Population

\begin{tabular}{|c|c|c|c|c|c|c|}
\hline \multirow{2}{*}{$\begin{array}{l}\text { LME Model } \\
\text { Fixed Effects } \\
\end{array}$} & \multirow[b]{2}{*}{$\underline{\text { Random Effects }}$} & \multicolumn{2}{|c|}{ PRx ARIMA Structure } & \multirow[t]{2}{*}{$\underline{\text { AIC }}$} & \multirow[t]{2}{*}{$\underline{B I C}$} & \multirow[t]{2}{*}{$\underline{\text { LL }}$} \\
\hline & & $\underline{p}$ & $\underline{q}$ & & & \\
\hline$P R x \sim S x \_a$ & intercept & 2 & 2 & -1543.672 & -1499.833 & 779.8362 \\
\hline PRx $\sim M x \_a$ & intercept & 2 & 2 & -1502.348 & -1458.509 & 759.1738 \\
\hline$P R x \sim S x \_a+M x \_a$ & intercept & 2 & 2 & -1550.704 & -1501.385 & 784.3520 \\
\hline PRx Sx_a & Intercept + Sx_a & 2 & 2 & -1564.957 & -1510.159 & 792.4768 \\
\hline PRx Mx_a & Intercept + Mx_a & 2 & 2 & -1516.367 & -1461.569 & 768.1836 \\
\hline$P R x \sim S x \_a+M x \_a$ & Intercept + Sx_a + Mx_a & 2 & 2 & -1597.345 & -1520.627 & 812.6726 \\
\hline
\end{tabular}

$A I C=$ Akaike Information Criterion, $A R I M A=$ auto-regressive integrative moving average, $B I C=$ Bayesian Information Criterion, $F V m=$ mean $T C D$ flow velocity, FVs = TCD based systolic flow velocity, ICP = intra-cranial pressure, $L L=\log$ likelihood, $L M E=$ linear mixed effects model, $p=$ auto-regression parameter for $A R I M A$ model, $M A P=$ mean arterial pressure, $P R x=$ pressure reactivity index (correlation between ICP and MAP), $q=$ moving average parameter for ARIMA model, Sx_a = systolic flow index (correlation between $T C D$ based FVs and MAP), TCD = transcranial Doppler. *Note: bolded value represents the most appropriate ARIMA structure and $L M E$ model for the patient population tested, based on principle of parsimony, lowest AIC and BIC. There was no integrative parameter (ie. " $d$ " parameter) included within the ARIMA models, given stationarity testing during patient examples (see appendix A and Methodology section of manuscript). 
Figure 1: Example of Recording Set up for ICP, NIRS and Robotic TCD

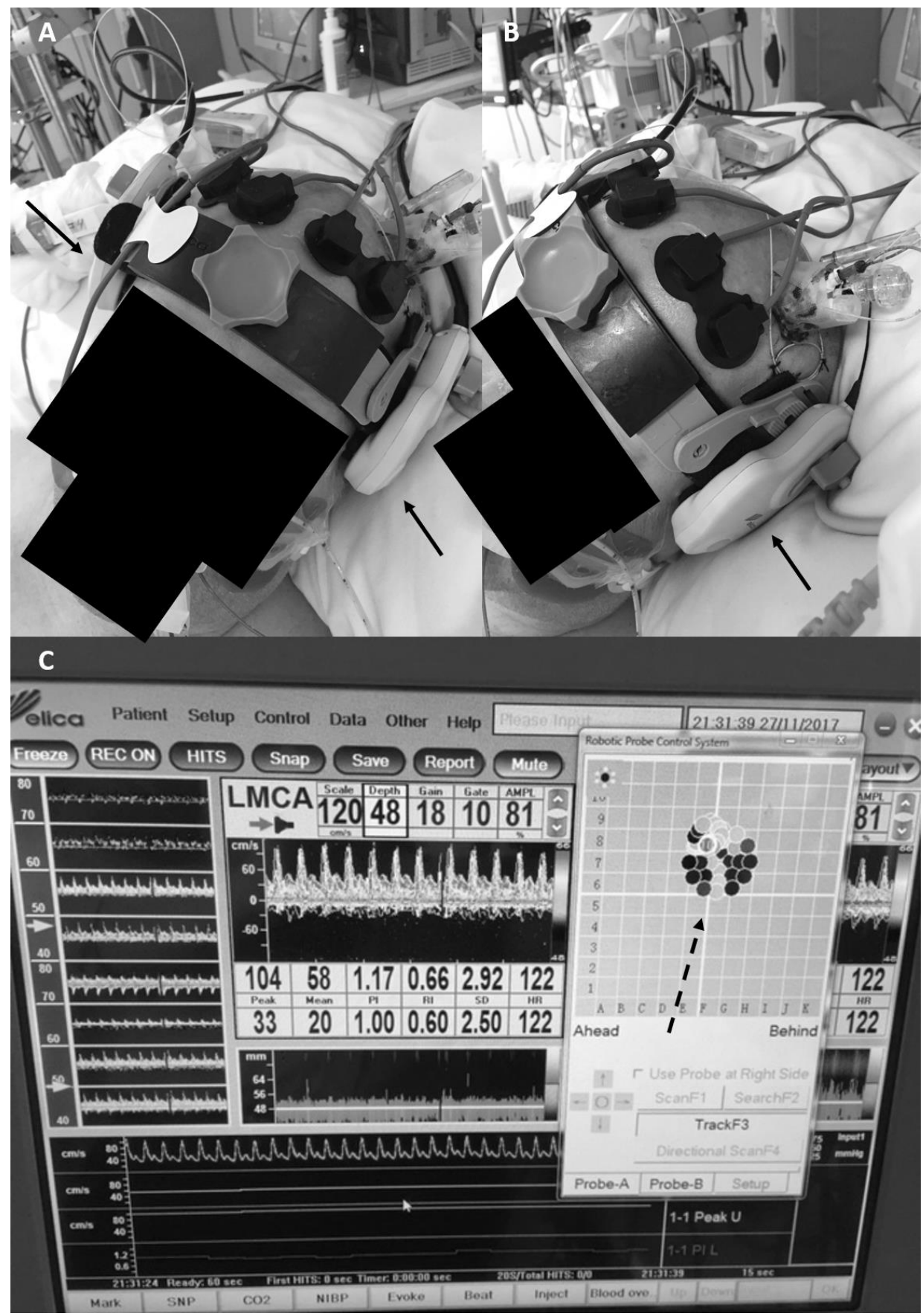

$I C P=$ intra-cranial pressure, NIRS = near infrared spectroscopy, $T C D=$ transcranial Doppler. Panel A: Displays left front bolt for ICP monitor, bifrontal NIRS pads (black bi-lobed pads on forehead), and robotic TCD secured with headband (black arrows denote the robotic drives which control TCD probe). Panel B: displays same as Panel A but from the side. Panel C: Delica EMS 9D TCD program display, showing TCD cerebral blood flow velocity wave form, M-mode display and automatic correction system (black hashed arrow - indicating the automated search pattern for the TCD correction algorithm, insonating at multiple sites, finding the area with superior signal quality). 

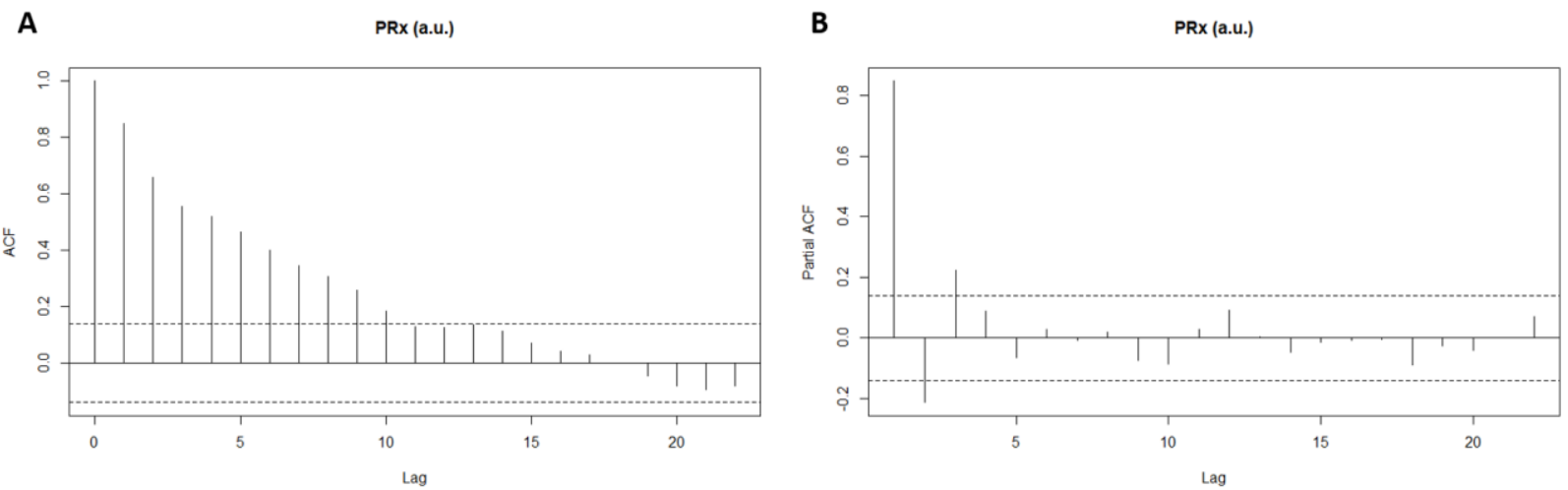

$A C F=$ autocorrelation function, a.u. = arbitrary units, $P A C F=$ autocorrelation function, $P R x=$ pressure reactivity index (correlation between intracranial pressure and mean arterial pressure). Panel A: ACF plot displaying a rapid decay of significant PRx lag, suggesting stationarity. Panel B: PACF plot, also displaying rapid decay of significant PRx lags.

Figure 3: Residual Plots for PRx (2,0,2) ARIMA Model and their ACF and PACF - A patient example

A

$(2,0,2)$ Model

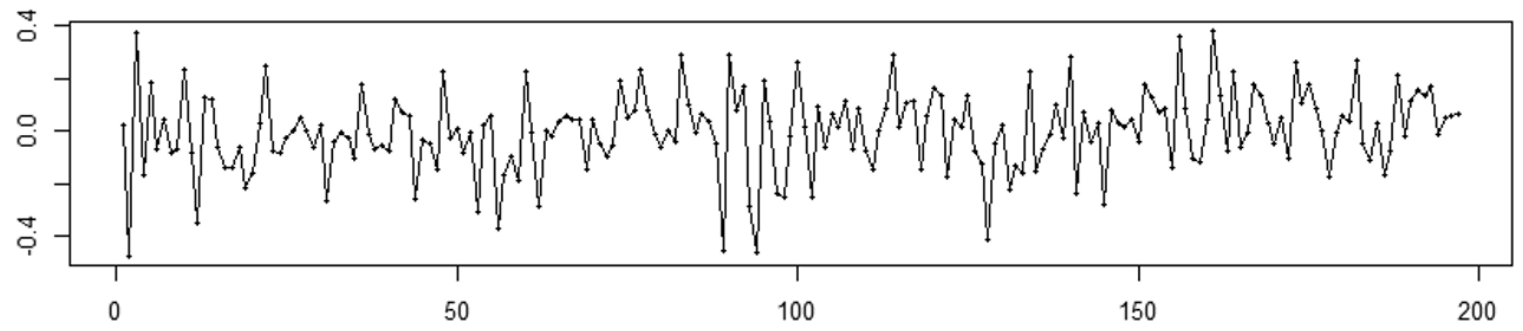

B

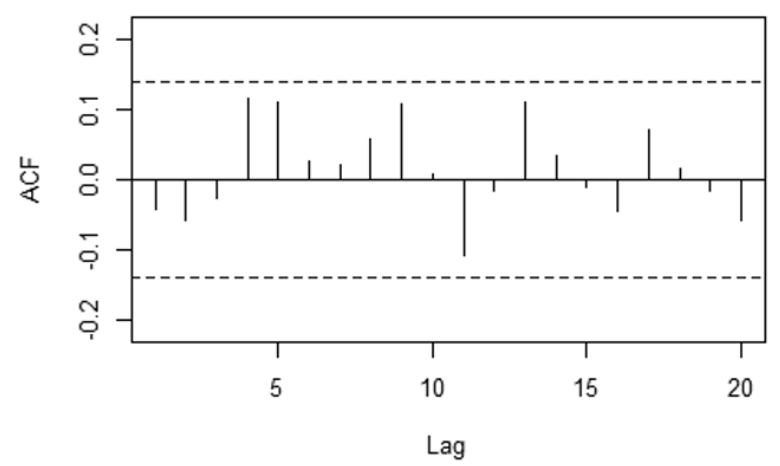

C

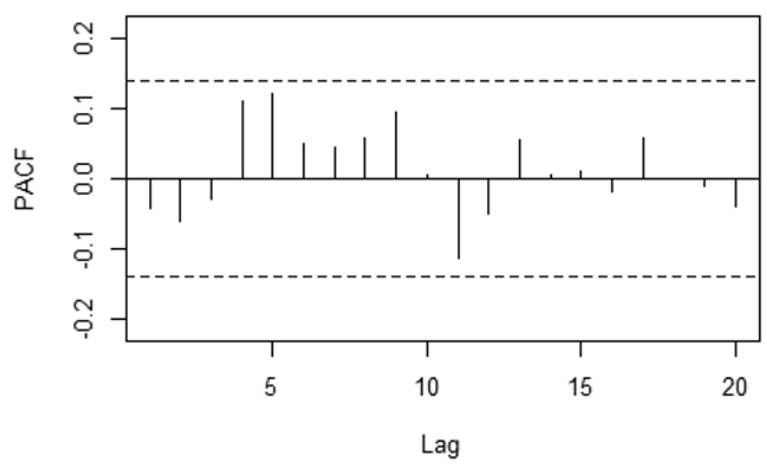

$A C F=$ autocorrelation function, $P A C F=$ autocorrelation function, $P R x=$ pressure reactivity index (correlation between intracranial pressure and mean arterial pressure). Panel A: displays the residual plot for the ARIMA model in this patient example. Panel B: ACF plot displaying no significant lags with $(2,0,2)$ ARIMA model for PRx. Panel C: PACF plot, also no significant lags with $(2,0,2)$ ARIMA model for $P R x$. 
Figure 4: Linear Regression Between Observed and Estimated PRx - Using Estimated PRx From Two Best LME Models
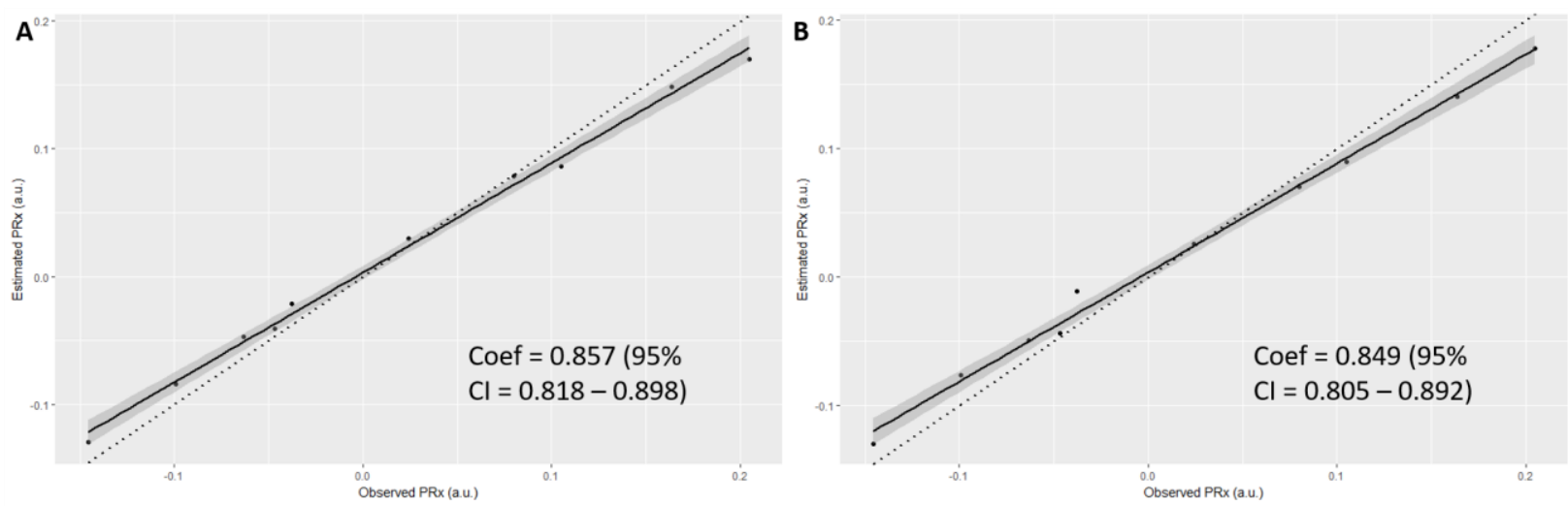

a.u. = arbitrary units, $I C P=$ intracranial pressure, $L M E=$ linear mixed effects, $M A P=$ mean arterial pressure, $P R x=$ pressure reactivity index (correlation between ICP and MAP). Panel A: LME model - PRx Sx_a (random effects with intercept and Sx_a), Panel B: LME model $-P R x \sim S x \_a+M x \_a$ (random effects with intercept, Sx_a and Mx_a). Coef = coefficients, form linear model between observed $P R x$ and model estimated PRx. Dotted straight line - represents the relationship " $y=x$ ", for comparison to our two models.

Figure 5: Linear Regression Between Observed and Predicted PRx - Using Predicted PRx From Two Best LME Models
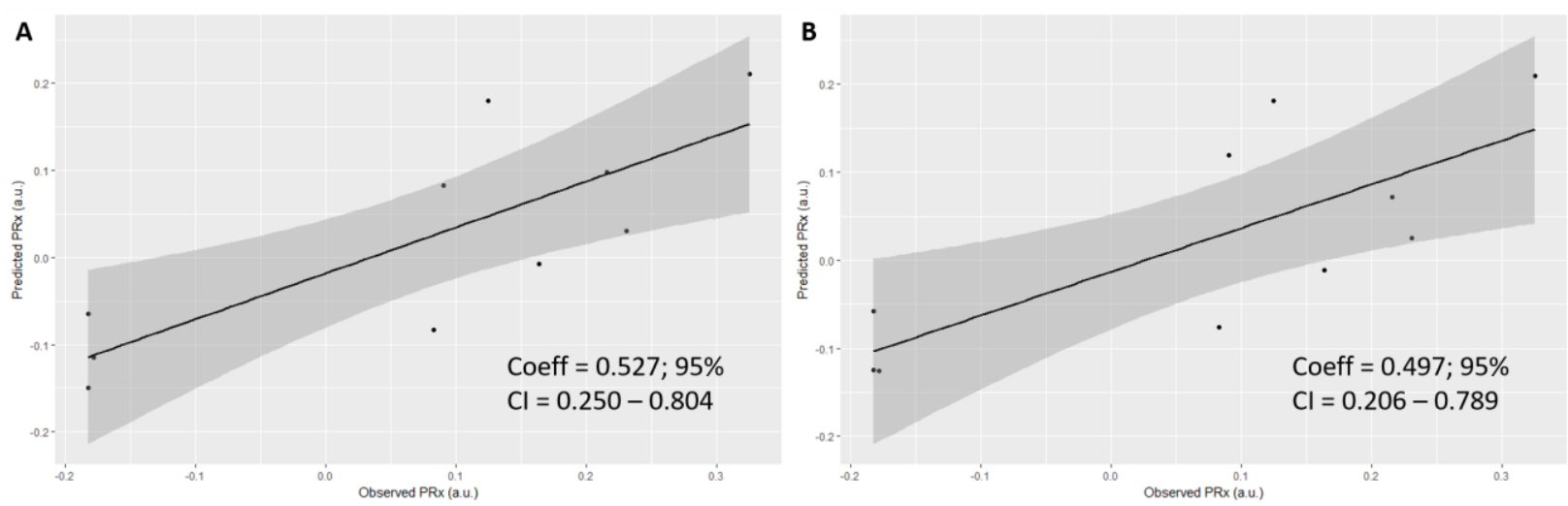

a.u. $=$ arbitrary units, $I C P=$ intracranial pressure, $L M E=$ linear mixed effects, $M A P=$ mean arterial pressure, $P R x=$ pressure reactivity index (correlation between ICP and MAP). Panel A: LME model - PRX Sx_a (random effects with intercept and Sx_a), Panel B: LME model $-P R x \sim S x \_a+M x \_a$ (random effects with intercept, Sx_a and Mx_a). Coef = coefficients, form linear model between observed PRx and model estimated PRx. 
Appendix A-QQ, Residual Density, ACF and PACF Plots for Top Two LME Models

1. PRx Sx_a LME Model (with random effects by patient in intercept and coefficients)

\section{QQ Plot}



\section{Residual Density Plot}

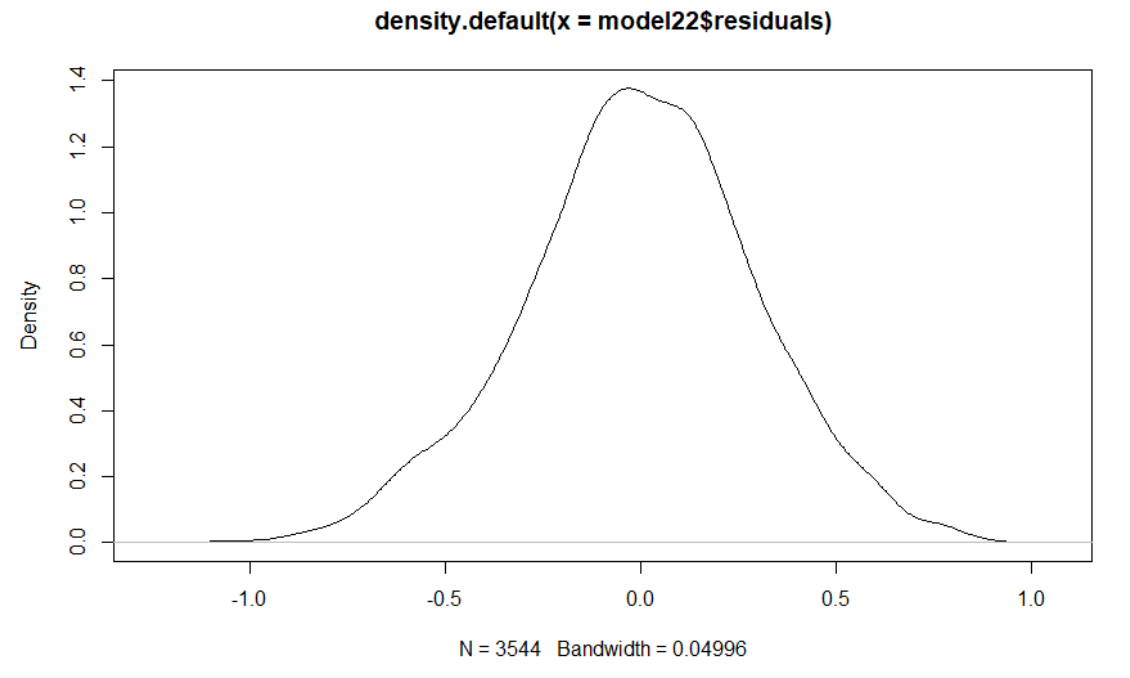


ACF Plot

Series residuals(model22)

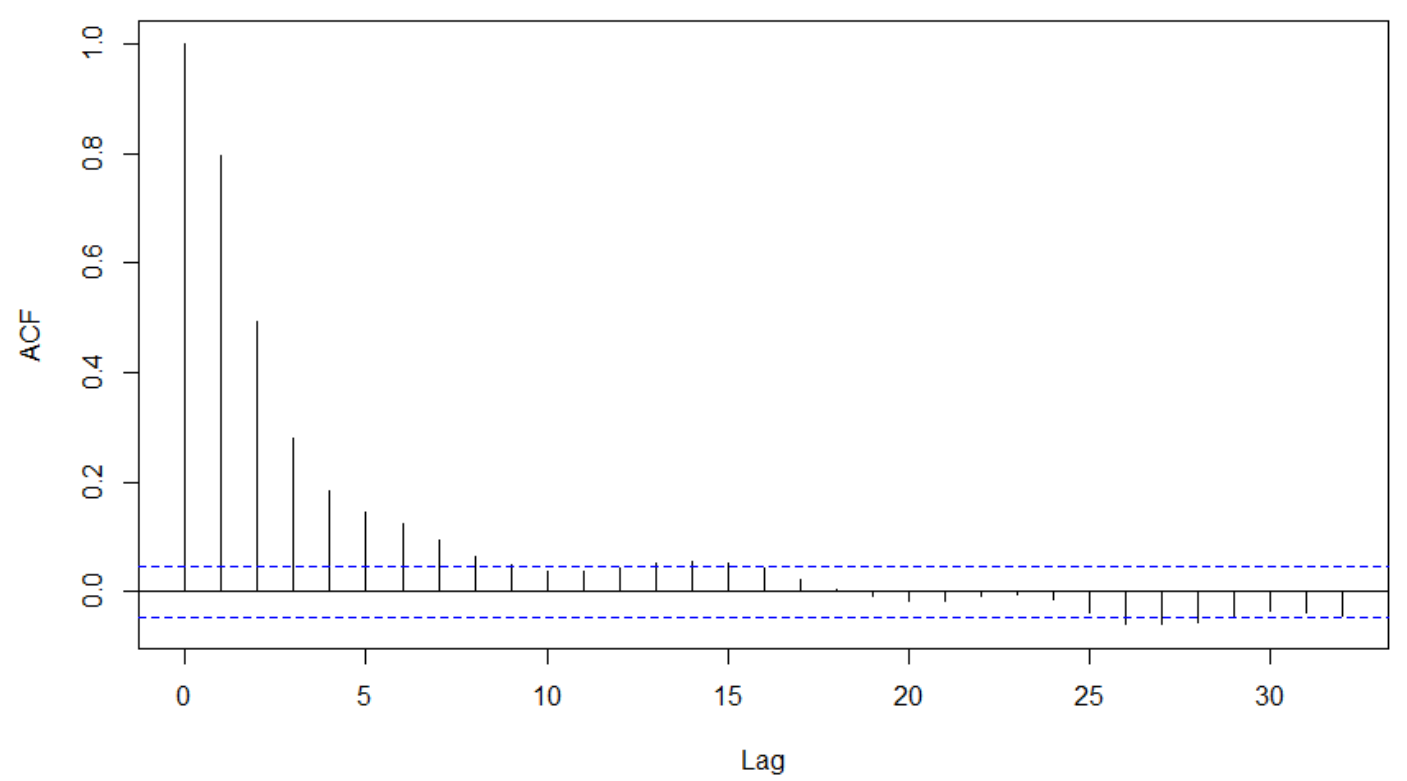

PACF Plot

Series residuals(model22)




2. PRx Sx_a + Mx_a LME Model (with random effects by patient in intercept and coefficients)

\section{QQ Plot}

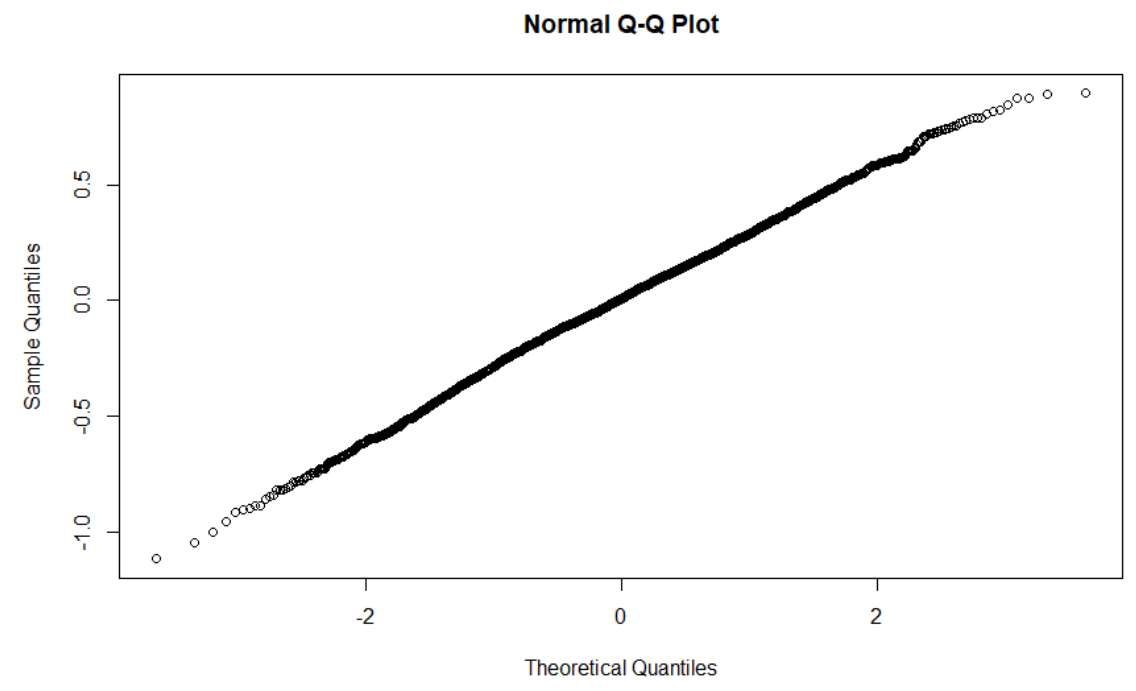

Residual Density Plot

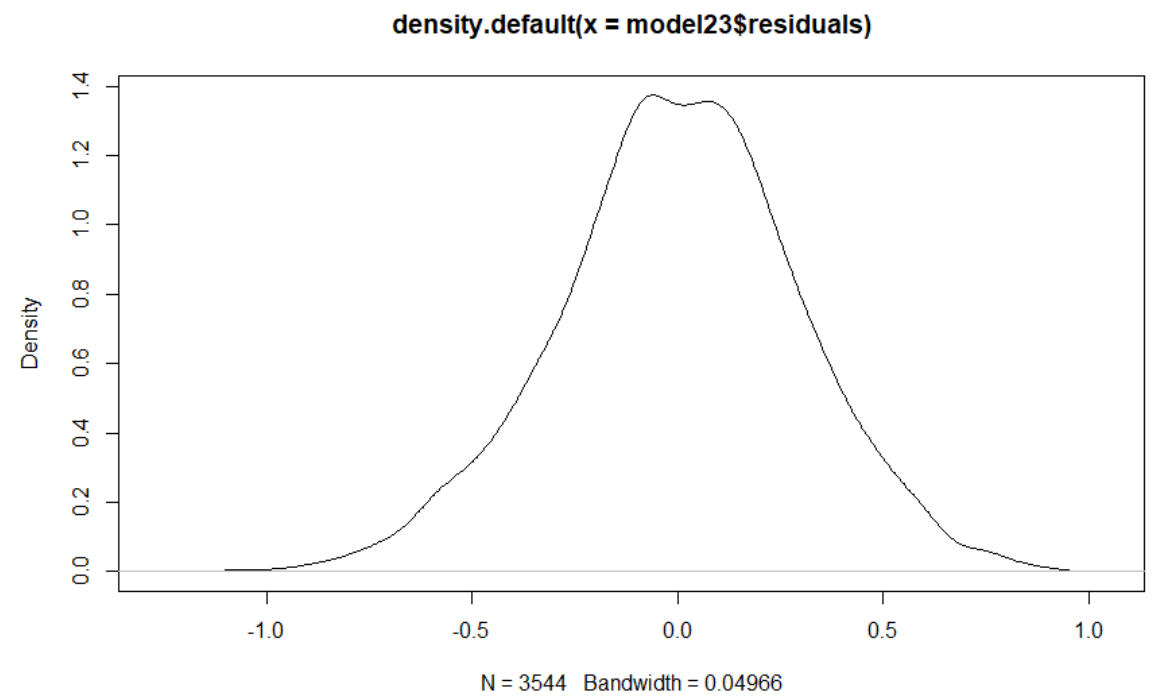




\section{ACF Plot}

Series residuals(model23)

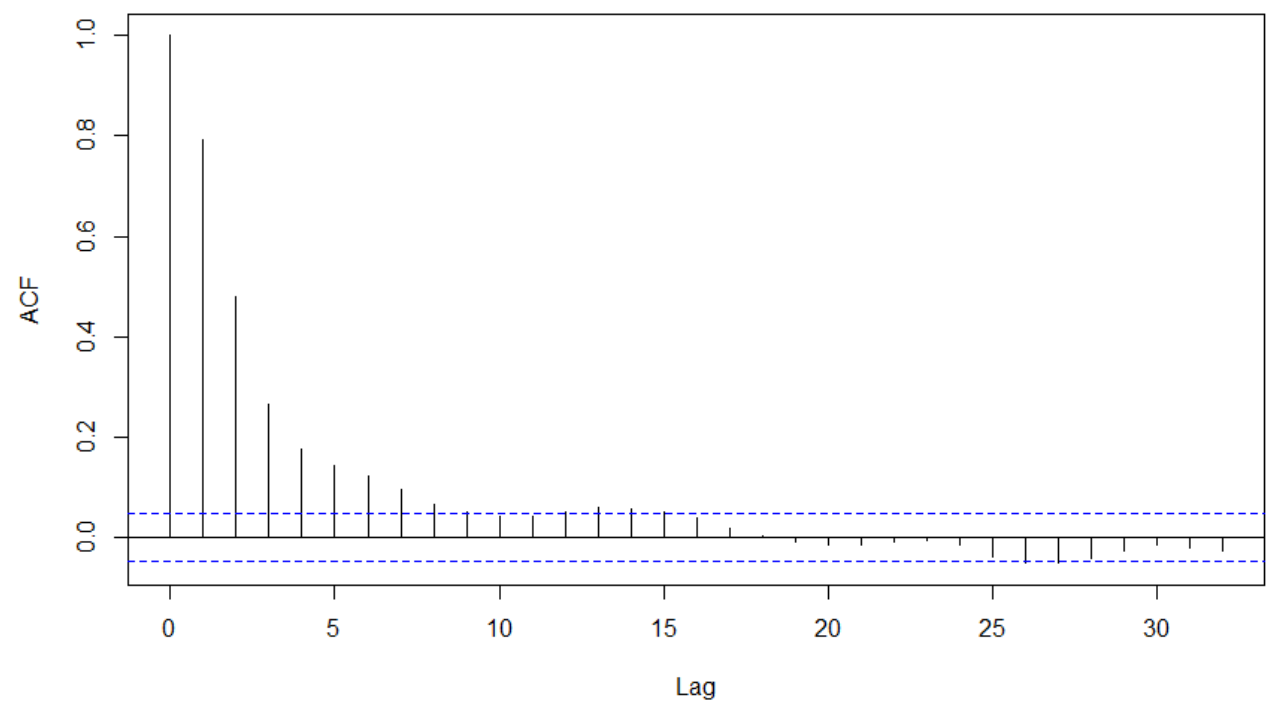

PACF Plot




Appendix B - Bland Altman Analysis for Estimated vs. Observed PRx for Top Two LME Models

*Grand mean Fisher transformed data utilized for Bland-Altman analysis

1. PRx Sx_a (with random effects introduced into intercept and coefficient)

\$1ower . 1imit

[1] -0.0628747

\$mean.diffs

[1] -0.003253664

\$upper. 1 imit

[1] 0.05636738

$\$ 1$ ines

lower.limit mean.diffs upper.7imit

$\begin{array}{lll}-0.062874705 & -0.003253664 & 0.056367377\end{array}$

\$CI. Tines

1ower. 1imit.ci.1ower 1ower.1imit.ci.upper

mean.diff.ci. 1ower

mean.diff.ci

.upper upper.7imit.ci.lower

$850671 \quad-0.10056477$

$-0.02518464$

$-0.02501403$

0.01

upper.1imit.ci.upper

0.09405744

\$critical.diff

[1] 0.05962104 


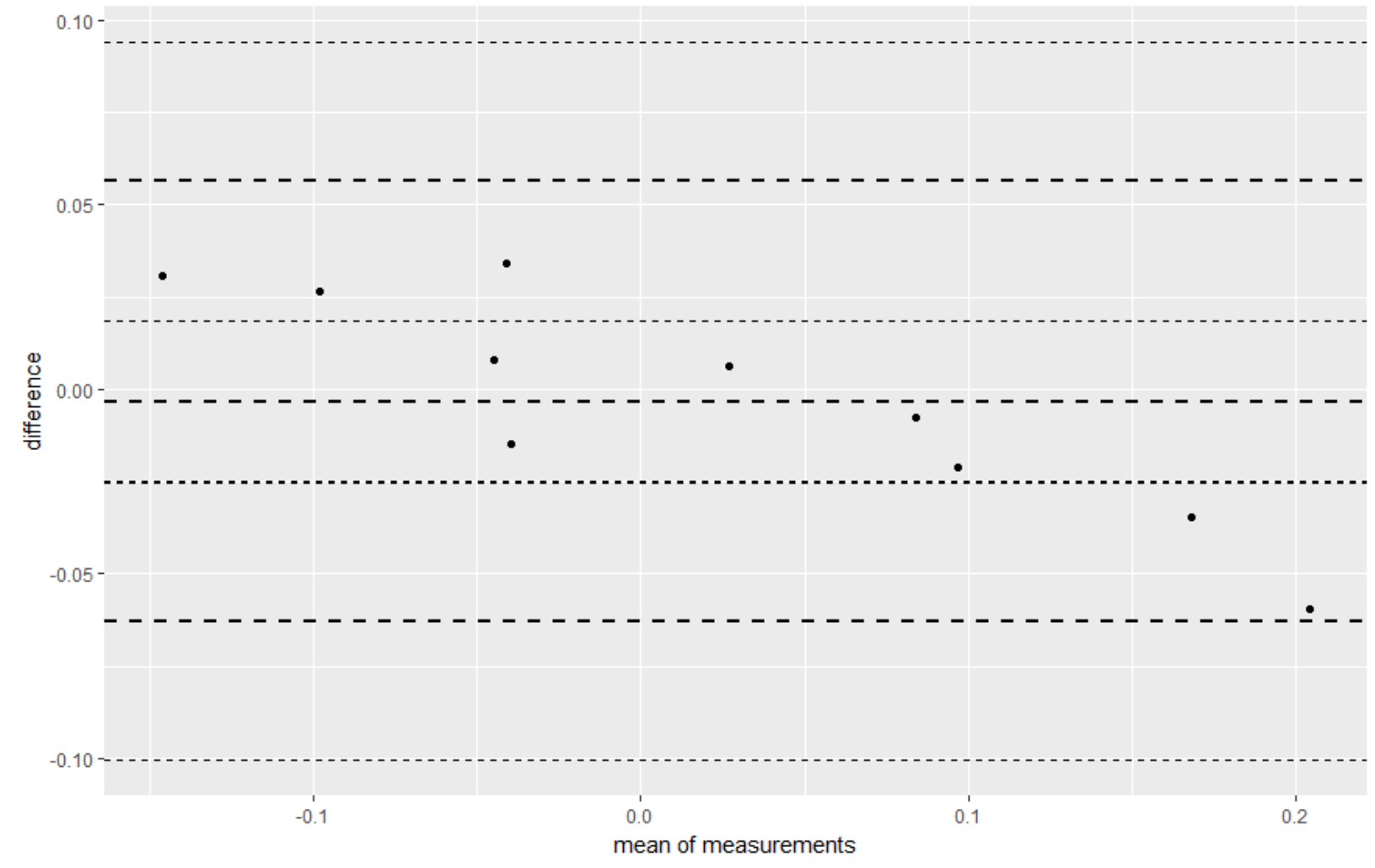

2. PRx Sx_a + Mx_a (with random effects introduced into intercept and coefficient)

\$1ower. 7 imit

[1] -0.0664208

\$mean.diffs

[1] -0.003062638

\$upper. 7 imit

[1] 0.06029553

$\$ 1$ ines

lower.7imit mean.diffs upper.7imit

$\begin{array}{lll}-0.066420801 & -0.003062638 & 0.060295526\end{array}$

\$CI. Tines

lower.limit.ci.lower lower.limit.ci.upper mean.diff.ci.lower mean.diff.ci .upper upper. limit.ci.lower

$\begin{array}{rr}006170 & -0.10647333 \\ 0.02024300\end{array}$

$\begin{array}{lll}-0.02636828 & -0.02618697 & 0.02\end{array}$

upper. Timit.ci.upper

0.10034805

\$critical.diff

[1] 0.06335816 


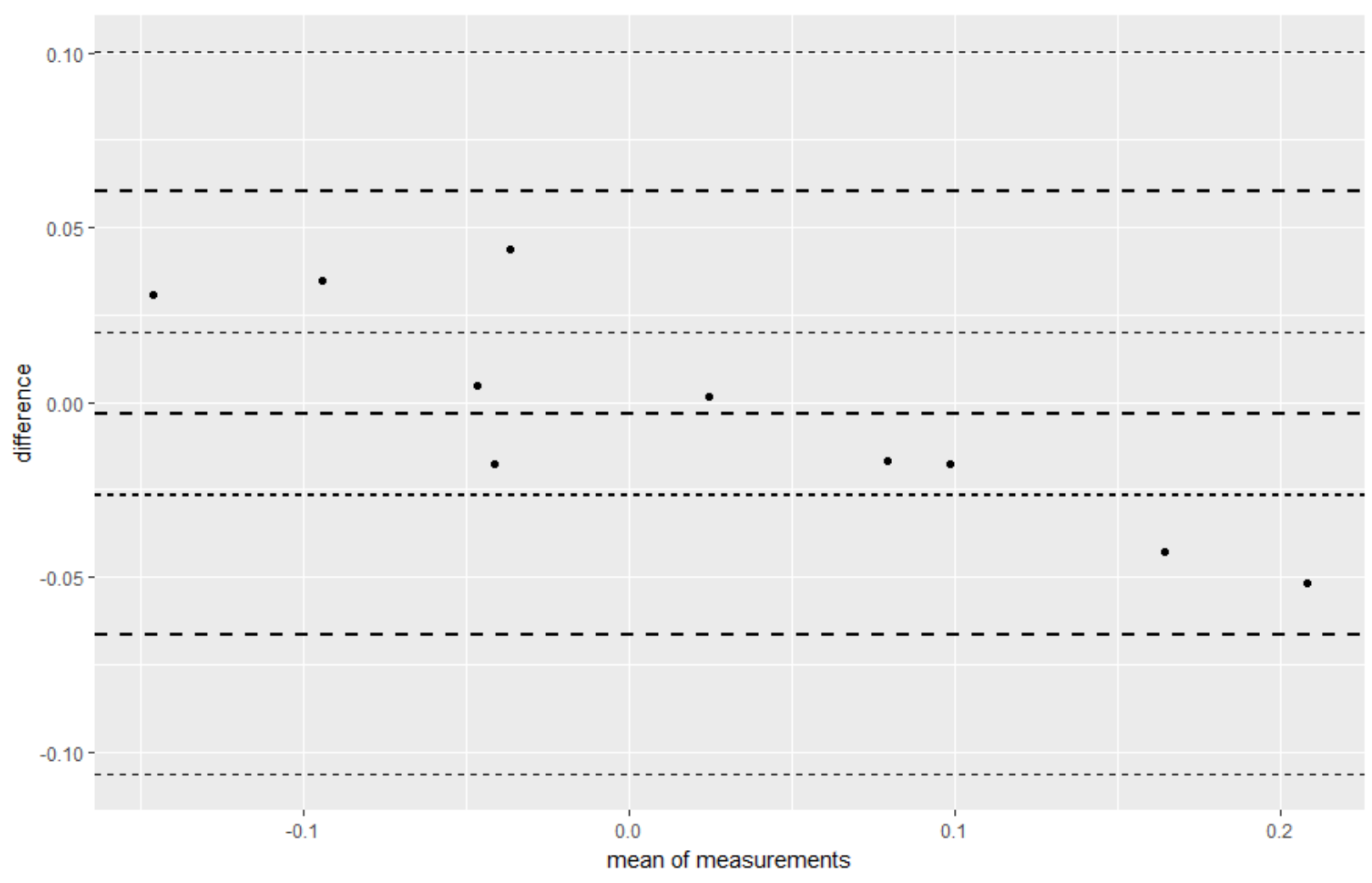


Appendix C-Bland Altman Analysis for Predicted vs. Observed PRx for Top Two LME Models

*Grand mean Fisher transformed data utilized for Bland-Altman analysis

1. PRx Sx_a (with random effects introduced into intercept and coefficient)

\$1 ower. 1imit

[1] -0.2826737

\$mean.diffs

[1] -0.05195699

\$upper. 1imit

[1] 0.1787597

\$1ines

lower.1imit mean.diffs upper.1imit

$\begin{array}{lll}-0.28267367 & -0.05195699 & 0.17875969\end{array}$

\$CI. 1ines

1ower. 1imit.ci.lower 1ower.1imit.ci.upper .upper upper. limit.ci.lower

$\begin{array}{cccc}2-0.42852364 & -0.13682371 & -0.13616351 & 0.03 \\ 224952 \quad 0.03290972 & & & \\ \text { upper.7imit.ci.upper } & & \end{array}$

\$critical.diff

[1] 0.2307167 


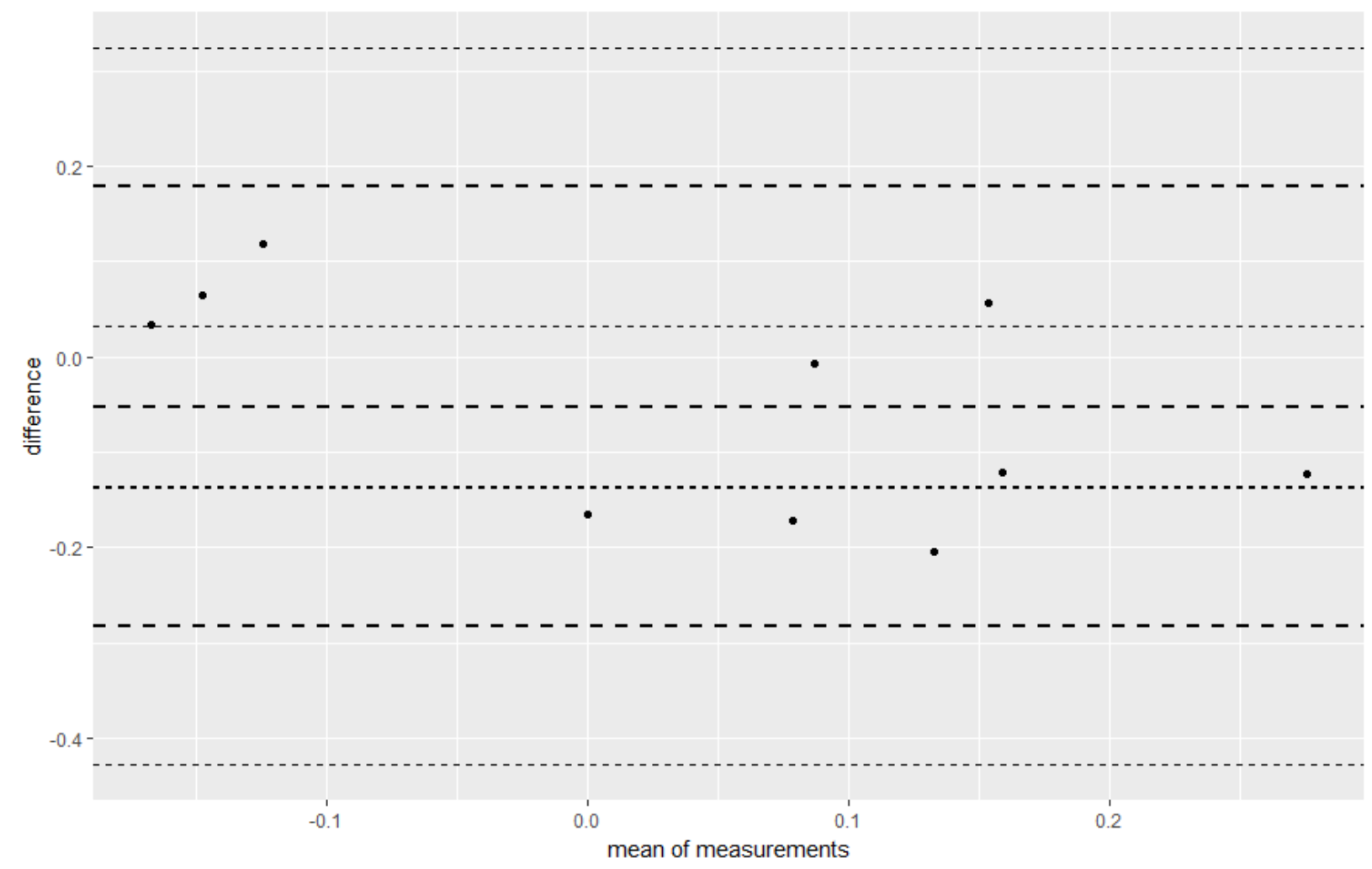

2. PRx Sx_a + Mx_a (with random effects introduced into intercept and coefficient)

\$lower. 7 imit

[1] -0.2932762

\$mean.diffs

[1] -0.04899657

\$upper. 1 imit

[1] 0.195283

$\$ 1$ ines

lower.7imit mean.diffs upper.7imit

$\begin{array}{lll}-0.29327618 & -0.04899657 & 0.19528303\end{array}$

$\$ C I .7$ ines

lower.limit.ci.lower lower.limit.ci.upper mean.diff.ci.lower mean.diff.ci .upper upper.limit.ci.lower

$016011 \quad-0.44770008$

$\begin{array}{lll}-0.13885227 & -0.13815326 & 0.04\end{array}$

upper. 1imit.ci.upper

0.34970694

\$critical.diff

[1] 0.2442796 


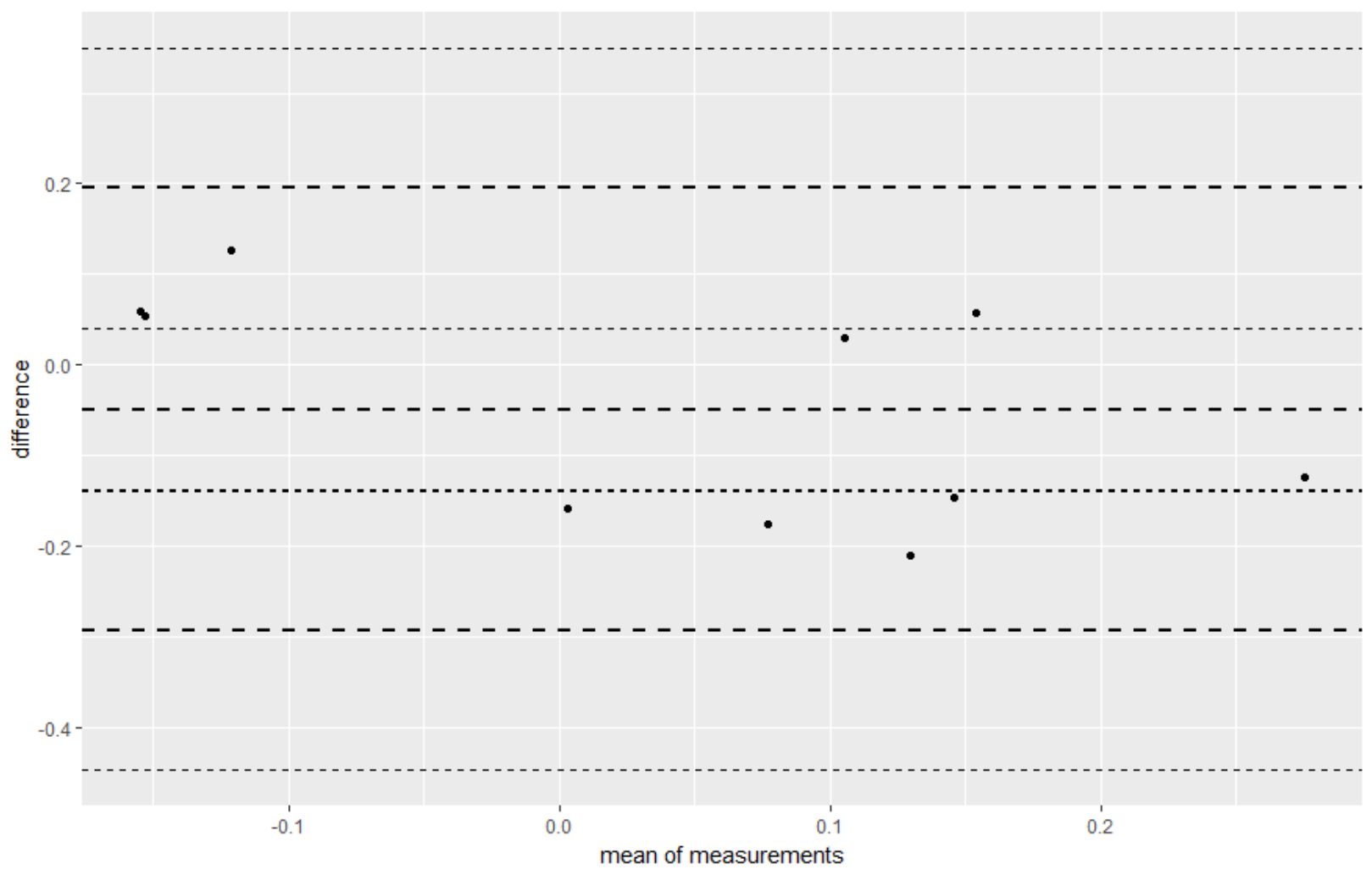

